\title{
ENSEIGNEMENT DU FRANÇAIS VIA L'HOMONYMIE DES TERMES MILITAIRES FRANÇAIS-RUSSES-LITUANIENS
}

\author{
Dr. Miroslav Stasilo
}

Vilniaus universitetas

\begin{abstract}
Sommaire. Cet article analyse l'enseignement et apprentissage du français langue étrangère dans l'Académie militaire de Lituanie en se focalisant sur la ressemblance et dissemblance de la terminologie professionnelle militaire en français, russe et lituanien. En se basant sur le corpus tiré de différents dictionnaires spécialisés, l'article présente la variété des usages des homonymes français dont l'emploi en lituanien et russe se différencie et s'associe en se focalisant sur l'étymologie et les valeurs sémantiques qu'ils acquièrent dans le discours militaire français-lituanien-russe moderne. Les résultats de cette recherche ont démontré que l'emploi des homonymes militaires coincent plus souvent en lituanien et en russe qu'en français et en lituanien. Cette étude a également présenté une grande complexité de l'homonymie dans le discours militaire actuel et son importante dans l'enseignement de français à l'académie.
\end{abstract}

Mots-clés: analyse lexicale, apprentissage, homonymie, discours militaire, termes militaires

\section{Introduction}

Le but de cet article est d'étudier et de présenter l'apprentissage du français langue étrangère (FLE) à l'Académie militaire de Lituanie du général Jonas Zemaitis (AML). Le processus éducatif y est basé sur un certain mélange de méthodes d'enseignement avec le choix pédagogique qui dépend de la situation didactique où l'étudiant collabore activement avec son enseignant. Cela nous permettra de toucher les trois objectifs : 1) comparer les méthodes traditionnelles et modernes d'enseignement du FLE aux élèves-officiers ; 2) présenter des particularités du discours militaire où l'homonymie occupe une place importante, selon nous ; 3 ) recueillir les exemples les plus illustratifs d'homonymes en français, russe et lituanien, auxquels nous avons recours fréquemment à l'AML. Pour cela nous nous sommes appuyés sur des méthodes descriptive, quantitative, qualitative et comparative dans nos recherches. Nous avons eu également recours aux œuvres d'Aristote, Ferdinand de Saussure, Charles Bally, Benjamin Bloom, Igor Vardul, Yuri Maslov, Stephen Ullmann, Evalda Jakaitiene et bien d'autres (cf. Bibliographie). Nos recherches se reposent également sur les traditions françaises de l'analyse du discours, notamment celles de Patrick 
Charaudeau et Dominique Maingueneau où le rôle du contexte est primordial.

Tous les exemples ont été pris des dictionnaires militaires spécialisés : «Terminologie militaire française 1670-1815 » de Donald Graves, «Dictionnaire de la réforme militaire » avec environ 2000 termes, crée par le Groupe de travail ad hoc du conseil d'OTAN et de Russie sur la réforme de la défense, " Dictionnaire des termes militaires » de l'OTAN. Mais les sources principales de mots recueillis sont : « Dictionnaire russe-français des termes militaires » (DRF), édité par l'université de Moscou sous la direction de Nikolai Garbovsky, «Lietuviu-anglu kalbu karo technikos žodynas » avec "Anglu-lietuviu kalbu karo technikos žodynas » d'Eugenijus Kisinas, «Enciklopedinis karybos žodynas » (EKZ), édité par AML, et « Tarptautiniu žodžių žodynas » (TZZ). De plus, le « Dictionnaire étymologique de la langue russe moderne » d'Aleksandr Shaposhnikov et le « Dictionnaire étymologique en ligne de la langue russe » de Max Vasmer ont été utilisés pour explorer l'étymologie des mots russes. Pour rechercher l'origine des termes français, nous avons consulté également le site du Centre National de Ressources Textuelles et Lexicales, qui nous donne un accès à la version informatisée du dictionnaire «le Trésor de la Langue Française » (CNRTL) ainsi que le dictionnaire Larousse (LR) et le site du dictionnaire lituanien des termes internationaux (DLTI).

\section{Homonymie}

Avant de présenter des exemples d'homonymes du discours militaire en français, russe et lituanien avec la comparaison des méthodes traditionnelles et modernes en enseignement du FLE à l'AML, il vaut mieux comprendre le phénomène d'homonymie qu'on rencontre souvent dans le discours militaire. Au début, nous voulons exposer la perception générale de l'homonymie.

C'est à noter qu'Aristote avait mentionné en premier le terme « homonyme » dans son œuvre Catégories : «On appelle homonymes les choses dont le nom seul est commun, tandis que la notion désignée par ce nom est diverse » (Aristote 2014, 8). Ensuite, d'après la conception de Ferdinand de Saussure, l'homonymie est perçue comme une situation où les mêmes signifiants peuvent avoir des signifiés différents (Saussure 1968). Cette idée est aussi mentionnée par Charles Bally, pour lequel les deux signes ayant les signifiants identiques et les signifies différents s'appellent les homonymes (Bally cité par Golovnja 2007, 17).

Selon plusieurs scientifiques, à savoir Igor Vardul, Yuri Maslov, Stephen Ullmann, l'homonymie peut exister sur plusieurs niveaux de la langue - de la morphologie à la syntaxe (idem, 10). De plus, selon le Petit Robert (PR), les homonymes sont les mots qui ont une écriture et une prononciation identique, mais des significations différentes (PR). Enfin, d'après Jakaitiene, ce sont les mots ayant la même expression et des significations complètement différentes (Jakaitiene 2009, 101).

Il y a un autre terme avoisinant à l'homonymie, c'est la paronymie. Les paronymes sont les mots dont la signification est différente, mais qui ont une pronon- 
ciation plutôt semblable grâce l'orthographe assez proche (Peciuliene, Simonaitis 2020). En d'autres termes, ce sont les mots ayant des signifiés différents et des signifiants voisins. Par exemple, les mots français « consommer» et « consumer $»$ : le premier nous renvoie au processus d'absorption d'un aliment ou d'une boisson, d'utilisation d'une matière comme source d'énergie, d'un produit alors que le deuxième verbe signifie « détruire peu à peu quelque chose en le réduisant à rien, spécialement par l'action du feu ou d'une très forte chaleur » (LR). Ce sont les paronymes car ils ont des sens différents mais s'écrivent et se prononcent d'une manière assez similaire.

Les points de vue sur le rôle d'homonymie dans la langue sont différents. Les uns pensent que c'est un enrichissement du vocabulaire, les autres trouvent les homonymes comme un phénomène négatif. Par exemple, Aleksandr Reformatsky et Aleksandr Gvozdev se classent parmi ceux qui considèrent l'homonymie comme un défaut de langage. Reformatsky a dit que « les homonymes ne jouent un rôle positif que dans les calembours et les anecdotes, où un simple 'jeu de mots' est nécessaire, dans d'autres cas, les homonymes ne sont qu'un obstacle à la compréhension » (Reformatsky 1996, 51). Leonid Bulakhovsky, Olga Akhmanova et Ruben Budagov sont d'accord que l'homonymie rend la compréhension de la parole difficile, mais ils n'ont pas de point de vue aussi négatif que les scientifiques mentionnés ci-contre. Les chercheurs ne parviennent donc pas à un consensus concernant l'évaluation de ce phénomène linguistique qu'on rencontre souvent dans le discours militaire.

\section{Discours militaire}

Selon le « Dictionnaire d'analyse du discours », tous les discours ont des caractéristiques de base : "Le discours suppose une organisation transphrastique (...) est orienté (...) une forme d'action (...) interactif (...) est contextualisé (...) est régie des normes (...) est pris dans un interdiscours » (Charaudeau, Maingueneau 2002, 185). Ces caractéristiques sont propres aussi au discours militaire : on y retrouve l'orientation vers les destinataires - militaires, susceptibles de comprendre le vocabulaire professionnel ; ce type de discours envisage une instruction ou formation professionnelle de ces allocutaires ; le traitement de ce discours n'est pas possible sans attention au contexte et aux liens avec d'autres formes de discours, par ex., le discours explicatif, argumentatif ou informatif car chaque texte militaire possède de l'explication, de l'argumentation et de l'information.

Dans le discours militaire, on présente et compare souvent le lexique professionnel, surtout dans les dictionnaires spécialisés qui appartiennent à ce type de discours. Tous ces dictionnaires sont nécessaires pour comprendre les textes spécialisés dans une langue étrangère. Cette partie du discours militaire est prioritaire pour nos recherches car elle est la plus recherchée par les étudiants qui étudient le français, surtout au début de l'apprentissage. 


\section{Apprentissage}

L'apprentissage, selon Benjamin Bloom (Bloom 1956), consiste à distinguer six niveaux d'ordre croissant chez l'apprenant qui acquiert un niveau donné à condition d'être capable de réaliser les opérations cognitives des niveaux inférieurs. Par exemple, si l'apprenant ne peut pas comprendre ni mémoriser (c'est le premier niveau des capacités intellectuelles), il n'accède pas au second niveau, celui d'application et d'analyse. Selon la taxonomie de Bloom, la capacité d'évaluer (synthèse et création) est le plus haut niveau des capacités intellectuelles chez l'apprenant (Grandmangin 2006, 30-31). Cela confirme l'idée que la mémoire et la compréhension sont recherchées en premier pour l'apprentissage.

Dans une démarche ancienne de grammaire-traduction, peu employée aujourd'hui lors de l'enseignement du FLE, on expliquait une règle de grammaire et on demandait à l'apprenant de l'apprendre par cœur, ensuite on faisait des exercices et on vérifiait si cette règle avait été bien comprise. Cela fonctionnait pour des exercices structurés puisque l'apprenant transmettait ses connaissances acquises sans garantir pourtant le même résultat dans une situation communicative. Les capacités de mémorisation, de compréhension et d'application étaient très sollicitées dans la méthode de grammaire-traduction alors que les niveaux supérieurs, comme l'analyse, la synthèse et la création, c'est-à-dire l'expression verbale, y ont été délaissés. Les méthodes qui se focalisent sur les approches communicatives et actionnelles font souvent appel à la capacité d'une analyse active dès le début de l'apprentissage puisque la réflexion personnelle est un facteur d'acquisition majeur dans la vie. On apprend mieux et plus profondément si on compare, réfléchit et observe des phénomènes linguistiques, ce qui nous amènent à la synthèse et à la création.

\section{Analogues phonétiques en français, lituanien et russe}

Avant de donner cinq exemples d'utilisation des homonymes lors de l'enseignement du FLE à l'AML, il est nécessaire de saisir les raisons de leur apparition grâce à une compréhension générale des relations entre les langues traitées. Le français, le lituanien et le russe appartiennent à la famille des langues indo-européennes dont les branches linguistiques sont différentes. La langue française est une représentante des langues romanes, le lituanien entre dans les langues baltes et le russe fait partie d'un très grand groupe de langues slaves. De plus, le russe et le lituanien sont des langues synthétiques, tandis que le français est une langue analytique, c'est-àdire qu'ils marquent les rapports grammaticaux différemment : le français n'a pas d'inclinaisons et son ordre de mots est stable alors que les deux autres langues ont des inclinaisons et une syntaxe assez libre. Par contre, l'alphabet français et lituanien est assez similaire alors que la langue russe possède un alphabet complètement différent : le français et le lituanien utilisent l'alphabet latin tandis que le russe - l'alphabet cyrillique. On voit donc que les trois langues sont à la fois différentes et semblables. 
En ce qui concerne l'histoire de l'apparition des homonymes dans ces trois langues, les emprunts français sont apparus dans la langue russe aux XVIII et XIX siècles grâce à la popularité du français à cette époque en Russie. Selon Jean Gerber, les mots empruntés sont enracinés dans la langue russe sous la forme et le sens de cette période, cela peut être aussi l'une des raisons de formation des homonymes français-russe (Gerber 2019). Par contre, nous n'avons pas trouvé de sources concernant l'apparition des homonymes français en lituanien. On sait seulement que les nouveaux emprunts, dont de la langue française, sont apparus massivement dans la langue lituanienne après la deuxième guerre mondiale (Girciene 2005, Vaicekauskienė 2007, Jakaitienè 2010).

Natalia Andrianova $(2009,4)$ avec Natalia Gabdreeva et Anastasia Ageeva $(2014,223)$ offrent des signes spécifiques du français dans la langue russe et nous y ajoutons leurs équivalents lituaniens. Cette liste sera utile lors de la sélection des homonymes pour notre analyse. Bien sûr, ce n'est pas une liste exhaustive, mais cela a été le point de départ de notre sélection des homonymes (cf. ci-dessous) :

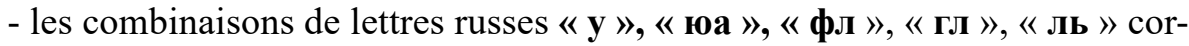
respondent à « $\mathbf{u} »$, « jua »; « $\mathrm{fl} »$, $\mathrm{gl} »$, , $\mathbf{l}$ » en lituanien et « ou », « oi », « $\mathrm{fl} »$, « gl », «l $\mathrm{l}$ en français ;

- les lettres initiales « a, э » sont des analoguse de «a, $\mathbf{e} »$ lituanien et «a,é $\gg$ français ;

- la fin des mots qui se termine par « аль », « ель », « $\mathbf{e p ~ » , ~ « ~ о н ~ » , ~ « ~ а н с ~ » , ~ « ~}$ ант », « аж », « » en lituanien et « al», « el », « ueur», « on », « ance », « ant», « age » en français ;

- les préfixes « ин », «де », « pe », « сюр », « дез » correspondent à « in », « de », « re », « sur », « dès » en français et « an », « de », « re », « siur », « dez » lituaniens ;

- les parties des mots-valises « арьер », « контр », « нон », « премьер », « гала », « пресс », « порт », « аван » sont associées en lituanien aux : « arjer », « kontr », « non », « premje », « gala », « pres », « port», « avan »; et « arrière », « contre », « non », « premier », « gala », « presse », « porte », « avant» en français.

Les trois langues n'ont pas que des ressemblances lexicales dont nous allons parler ci-bas mais aussi phonétiques - il y a plein de mots qui ont l'air ou se prononcent d'une manière semblable, mais qui signifient des choses différentes.

\section{Homonymes dans le processus d'apprentissage}

Les apprenants de l'AML, qui étudient le FLE, s'appuient sur plusieurs méthodes dont le but principal est leur formation professionnelle en français. Ils ont besoin par conséquent de maîtriser un vocabulaire spécifique qu'on retrouve dans le discours militaire. Nous allons présenter ici seulement cinq homonymes à cause des règles du journal pour le nombre de pages. Ce sont les vocables, selon nous, les plus employés et assez illustratifs dans le contexte actuel de l'apprentissage dans 
l'académie. Notre présentation des échantillons lexicaux se base sur un caractère alphabétique français. Le premier exemple, c'est un mot très employé en français :

(1) « appareil » - аппарель » (ru), « apparele » (lt)

Le terme « appareil » signifie : « ensemble d'éléments qui concourent au même but en formant un tout », " assemblage de pièces ou d'organes (plus complexe que l'outil, l'ustensile, moins que la machine) réunis en un tout pour une fonction » (CNRTL).

La fin de ce mot français « -eil » nous renvoie aux terminaisons semblables (cf. ci-dessus) - « -ель » en russe et « elè » en ltuanien. On a donc « аппарель » russe et « apparelè » lituanien. Ce sont les homonymes qui s'écrivent et se prononcent presque de la même manière, mais signifient des objets différents. Le mot russe « аппарель » est venu de la langue française et cela signifie aujourd'hui en russe « une ascension douce vers les parties élevées des bâtiments, des structures ou une pente douce vers quelque chose, la plateforme inclinée pour le chargement et le déchargement des équipements » (Kuznecov, 2014). En lituanien, la signification de ce vocable est proche à celle en lituanien : "nuožulni iškasa apkaso ar priedangos šlaite kovos technikai ịvažiuoti » (EKZ). On voit donc que c'est un équipement avec un mécanisme qui devrait être monté et qui a une fonction particulière. Il est important à noter, que ce mot n'est utilisé en russe et en lituanien que dans le discours militaire, alors que dans l'architecture des structures civiles, on emploie d'autres vocables : "prietaisas, platforma, rampa» (lt), « устройство, платформа, спуск » (ru).

Selon les dictionnaires bilingues des termes militaires, si l'on rencontre les mots « аппарель » $(\mathrm{ru})$ et « apparele » (lt), il faut les traduire en français comme « rampe d'accès à la cale », " plan incliné de chargement » (CNRTL). Après la recherche dans plusieurs sources, entre autres, «Dictionnaire étymologique de la langue russe moderne », «Dictionnaire étymologique en ligne de la langue russe ", "Lietuviu-anglu kalbu karo technikos žodynas » et « Anglu-lietuviu kalbu karo technikos žodynas ", nous ne pouvons pas définir le moment exact de l'apparition de cet homonyme français ni en russe ni en lituanien. Aussi, nous pouvons faire l'hypothèse qu'on a commencé à employer ce terme français d'une manière facultative en appliquant le schéma suivant - partie au lieu d'entier, c'est-à-dire, on a réduit le sens initial en appelant une espèce au lieu de tout le groupe.

Voici un autre exemple qui est assez fréquent en russe et que les élèves-officiers de Lituanie ont tendance à mélanger avec d'autres termes militaires :

(2) « as » - « ac » (ru) et «asas, ąsa» (It)

Le mot français « as » est apparu en France au XIIème siècle pour désigner « une figure, point unique que l'on rencontre sur une carte, un côté de dé ou une moitié de domino (...) carte, côté de dé, moitié de domino possédant une figure ou un point unique » (CNRTL). Ce terme est un emprunt du latin, où le vocable désignait une pièce de monnaie que les Romains utilisaient dans leurs échanges commerciaux (idem). Et c'est à partir du début du XXème siècle que ce mot commence à être 
employé pour appeler quelqu'un qui « par sa valeur, détient le premier rang dans le domaine militaire, notamment dans l'aviation de chasse (...) personne généralement du sexe masculin excellant dans un domaine » (idem).

En russe moderne, cet homonyme signifie un " pilote de chasse ayant une performance exceptionnelle en vol et en combat. Un grand maître, un excellent spécialiste dans son domaine » (DRF). Le dictionnaire confirme, que ce mot russe « ac » est un emprunt du français. Dans ce cas-là, le mot russe est monosémique et le mot français, dont l'une de ces significations françaises a été transférée au russe, est polysémique. La signification du mot français « as " est en relation homonymique d'équivalence avec le vocable russe « ac» dans le contexte de jeux. Pour la référence seulement, les Russes appellent « туз » (joker en fr.) pour nommer un « as » dans un jeu de cartes.

Quant à la langue lituanienne, il y a deux versions de ce mot français - «asas » et « ąsa ». Le premier signifie « labai geras savo specialybès žinovas, meistras (lakūnas, sporto lyderis, dainininkas » (DLTI) et le second - «viena iš jungiamuju daliu : diržo tvirtinimo, kablio, keitiklio svirties » (Kisinas 2015, 26). On aperçoit que la première signification est proche à la langue russe où ce mot est associé à une personne professionnelle de plusieurs domaines, notamment en aviation, alors que la deuxième désignation est un terme spécial dont l'emploi est très limité. Comme la prononciation lituanienne de ces deux mots est assez semblable, les étudiants lituaniens oublient souvent l'existence des deux significations différentes.

Deux autres vocables ne sont pas les termes pures militaires même s'ils sont assez présents dans le discours militaire actuel français, russe et lituanien :

(3) « benzine » - « бензин » (ru), « benzinas » (lt)

Selon le dictionnaire du Centre National de Ressources Textuelles et Lexicales, le mot « benzine » signifie en français « liquide incolore, d'une odeur d'éther, à base de benzène, utilisé comme détachant ou dissolvant », mais avant, c'était utilisé comme un « carburant pour moteurs à explosion » (CNRTL). En russe, le mot « бензин » signifie l'essence, au sens du carburant. En lituanien, ce vocable a la même signification qu'en russe : «Bespalvis, lengvai garuojantis ir užsiliepsnojantis skystis (...) Būna automobilinis ir aviacinis » (EKZ). L'analyse étymologique de ce vocable indique son apparition en russe et en lituanien grâce au français. On voit par conséquent que l'emploi archaïque et l'origine française de cet homonyme se ressemblent en russe et en lituanien d'où est la conclusion que c'est la juxtaposition du sens archaïque contre le sens contemporain dans les langues différentes (Tomilova, 2018).

Les deux autres termes ne sont employés que dans la vie et le discours militaires. C'est pourquoi ces homonymes sont très importants pour la formation professionnelle en français. Le premier mot est :

(4) « blindage » - « блиндаж» (ru), « blindažas » (lt)

La fin «-age » a attiré notre attention car elle rappelle l'une des terminaisons françaises les plus fréquentes à côté de «-tion », «-sion » et «-isme », qui prouvent 
l'origine française des mots dans d'autres langues. Le suffixe français « -age » se transforme en «-аж» dans la langue russe et «-ažas » en lituanien (cf. ci-dessus). Selon le dictionnaire russe Большой толковый словарь русского языка (BTSR), le terme « блиндаж », c'est « une construction défensive, un approfondissement dans le sol avec un revêtement de bûches pour se protéger contre l'artillerie, le mortier, tir d'ennemi » (BTSR). Il est indiqué que ce mot russe vient du français. Selon le dictionnaire français de l'Académie française, le mot «blindage » signifie plutôt une action, " c'est une action de blinder », " un revêtement protégeant un véhicule des projectiles », « dispositif recouvrant un objet pour le protéger, l'isoler » (DAF).

Le dictionnaire lituanien des termes militaires donne la signification suivante de ce vocable spécialisé : " uždarasis lauko fortifikacinis ịrenginys 4-8 kariams. Turi tvirta dengini (...) Pirma karta buvo įrengti Rusijoje per Sevastopolio gynyba 1854-1855. Buvo naudojami per ir I ir II pasaul. karus. 》 (EKC) On observe ici non seulement la ressemblance de l'emploi avec la langue russe où ce substantif est associé à une « construction défensive » mais aussi l'origine et le lien avec l'histoire russe - la défense de la ville de Sevastopol en Crimée et deux Guerres Mondiales. C'est par ailleurs un cas assez fréquent dans la langue lituanienne quand les termes militaires d'origine française apparaissent dans le vocabulaire professionnel de Lituanie via la langue russe. Nous avons de même remarqué que les apprenants de l'AML retiennent plus facilement ce type de mots que les vocables qui se retrouvent directement du français, par ex., « as »(cf. ci-dessus).

On voit que les mots français qui correspondent le mieux aux termes russes et lituaniens ici sont : un abri de combat renforcé, un blockhaus. Pour catégoriser ce mot dans l'une de nos catégories, on peut noter ainsi que ces termes sont du même domaine - ils sont liés à la protection. Cependant, le degré de leurs sens est diffèrent - le mot français est plus général, alors que les vocables russes et lituaniens ont un sens plus spécifique, étroit, c'est-à-dire, on assiste à une relation d'inclusion où les substantifs « блиндаж » russe et «blindažas » lituanien sont des hyponymes de leur hyperonyme français « blindage ». (CNRTL)

Voici notre dernier exemple d'analyse qu'on emploie souvent aux cours de français à l'AML. A noter que son utilisation en lituanien et russe se ressemble aussi :

(5) « camouflage » - « камуфляж» (ru) « kamufliažas » (lt)

La terminaison «-яж» du mot russe « камуфляж» témoigne de ses racines françaises et cela est confirmé par le grand dictionnaire russe Большой толковый словарь русского языка. En russe, dans le domaine militaire, c'est une méthode de camouflage, qui consiste à peindre des fusils, des navires, des bâtiments aux taches, rayures déformant leurs contours et en les rendant difficiles à détecter (BTSRY). Aussi, ce qui est utilisé pour un tel déguisement (DERE). Au sens figuré, ce vocable signifie des actions, des déclarations servant à cacher la vraie nature des objets (idem). Dans le langage quotidien, les Russes utilisent ce mot pour appeler les uniformes de couleur tachetée grises et vertes (DEEK) et un tissu teint avec des 
taches de brun, verdâtre ou gris, blanc et d'autres taches employées pour les vêtements paramilitaires (DSES). On appelle « камуфляж» des vêtements faits d'un tel tissu (DEO). En russe, la capacité inhérente de certains animaux à prendre la couleur d'environnement peut aussi être appelée « камуфляж» (DEEK).

Le mot français « camouflage », c'est « une action de camoufler ou un résultat de cette action » (CNRTL). Dans le domaine militaire, c'est « un ensemble de procédés permettant de masquer ou de dissimuler, aux yeux de l'ennemi, des troupes, du matériel de guerre, des positions de campagne » (DAF) « en utilisant des moyens naturels ou artificiels » (CNRTL). Au sens figuré, c'est un artifice par lequel on déguise la réalité; c'est une fraude, tromperie (CNRTL). Le mot, apparu au XIXème siècle, est un dérivé du verbe " camoufler » qui a son tour est originaire du nom « camouflet» (la notion de 'dissimulation' étant issue de celle de 'fumée') (DAF). De plus, depuis le XXème siècle, les Français utilisent le mot en parlant des télécommunications : « camouflage d〉un message radio, téléphonique » (idem), ainsi que du théâtre : " tout ce qui concerne la transformation complète d'un acteur pour l'interprétation d'un rôle : costume et maquillage savamment combinés » (idem).

En lituanien, le vocable « kamufliažas » n'appartient qu'au discours militaire car il signifie " karo lèktuvu, laivu, tanku, pabūklu dažymas stambiais dryžiais, dèmèmis, kad ju kontūrai atrodytu kitokie ir priešininkui būtu sunku juos atpažinti » (TZZ). Si on veut utiliser ce substantif pour d'autres type de discours, le dictionnaire anglais-lituanien nous propose d'employer plutôt le mot lituanien «maskuité, maskavimas, priedanga » (Kisinas 2013, 59). Ainsi, il y des significations russes, françaises et lituaniennes qui coïncident mais il y en a également celles qui sont spécifiques autant pour le russe ou le lituanien, comme «tissue et vêtements » et " capacité inhérente de certains animaux », que pour le français dans le domaine de télécommunications et du théâtre. C'est un résultat d'évolution sémantique des mots ayant dès le début des zones sémantiques proches et éloignés. Selon le classement de Garbovsky (Garbovsky, 2007), les mots « camouflage » et « камуфляж », « kamufliažas» sont donc dans une relation de l'intersection.

Avant de terminer cet article, il faut mentionner qu'on retrouve plus d'homonymes dans les dictionnaires mentionnés dans l'introduction (cf. ci-dessus). On peut y voir également d'autres cas qui nous paraissent assez fructueux pour des recherches possibles postérieures.

\section{Conclusions}

Dans cette étude, nous nous sommes intéressés à l'emploi des homonymes pendant le processus d'apprentissage à l'AML où les méthodes traditionnelles d'enseignement, comme la traduction des textes professionnelles en français vers le lituanien, par exemple, sont parfois à côté des méthodes innovatrices, par exemple, le travail à distance. L'utilisation des homonymes y est possible grâce à leur émergence dans le discours militaire français-lituanien-russe. Les exemples présentés ci-dessus 
nous ont permis de voir les cas dans lesquels ces homonymes sont assez proches, surtout en lituanien et russe. Ce sont également les vocables que les apprenants lituaniens retiennent et emploient plus facilement dans les cours de français à l'AML en évitant cependant l'utilisation des homonymes lexicalement assez éloignés. De plus, nous avons compris que l'homonymie française - russe - lituanienne n'est pas un phénomène simple, mais très complexe, ayant plein de choses à explorer. L'émergence d'homonymes dans ces trois langues a été influencée par plusieurs facteurs comme les événements historiques, l'influence d'autres langues, les processus de développement économique et politique, ou parfois une simple coïncidence.

\section{Bibliographie}

Aristote. Traduction de J. Tricot (2014). Catégories. Paris : Editions Les Echos du Maquis

Bloom, B. S. (1956). Taxonomy of Educational Objectives. Boston: Published by Allyn and Bacon

Charaudeau, P., Maingueneau, D. (2002). Dictionnaire d'analyse du discours. Paris

Girčienè, J. (2005). Naujujų skolinių atitikmenys: struktūra ir vartosena. Vilnius

Grandmangin, M. (2006). La classe de langue. Paris : CLE international

Jakaitiené, E. (2010). Leksikologija. Vilnius

Jakaitiene, E. (2009). Leksikologija. Studijų knyga. Vilnius: Vilniaus universiteto leidykla

Peciuliene, O., Simonaitis, R. (2020). Paronimai. Vilnius : Mokslo ir enciklopedijų leidybos centras

Saussure, F. d. (1968). Cours de linguistique générale. Paris: Editions Payot

Vaicekauskienè, L. (2007). Naujieji lietuvių kalbos svetimžodžiai. Vilnius

Андриянова, Н. (2009). Военная и научно-техническая терминология французского происхождения в современном русском языке. Автореферат

Габдреева, Н., Агеева, А., (2014). Русско-французские языковые контакты: Процесс заимствования и ассимиляции как следствие межкультурной коммуникации. Коммуникативные исследования № 1, 221-232

Гарбовский, Н. (2007). Теория перевода. Издательство Московского университета

Головня, А. (2007). Омонимия как системная категория языка: монография . Minsk: БГУ

Жербер, Ж. (2019). Филологический класс. № 3 (57). - 65-72

Реформатский, А. (1996). Введение в языковедение/Под ред. В.А Виноградова. Аспект Пресс 


\section{Dictionnaires}

CNRTL. (2012). Trésor de la Langue Française. Etymologie. Centre National de Ressources Textuelles et Lexicales. Consulté le 14 juin 2021, site internet: http:// www.cnrtl.fr/etymologie/B\%C3\%A2ton

Enciklopedinis karybos žodynas (2008). Vilnius: Generolo Jono Žemaičio Lietuvos karo akademijos leidykla

Harper, D. (2020). Online Etymology Dictionnary. Lancaster, PA, US. Consulté le juin 2, 2021, site internet https://www.etymonline.com/word/compass

Kisinas, E. (2013). Anglų-lietuvių kalbų karo technikos žodynas. Vilnius: Generolo Jono Žemaičio Lietuvos karo akademijos leidykla

Kisinas, E. (2015). Lietuvių-anglų kalbų karo technikos žodynas. Vilnius: Generolo Jono Žemaičio Lietuvos karo akademijos leidykla

Larousse. (2020). dictionnaire de français. Consulté le mai 4, 2021, sur larousse.fr: https://www.larousse.fr/dictionnaires/francais

Robert (2020). Le Robert dico en ligne. Consulté le mai 10, 2021, sur https:// dictionnaire.lerobert.com/definition/bilingue\#definitions

Tarptautinių žodžių žodynas (2013). Vilnius

Большой словарь иностранных слов русского языка (2019). Consulté le 14 février 2019, site internet: https://gufo.me/dict/foreign_words/\%D0\%B4\%D $0 \% \mathrm{~B} 5 \% \mathrm{D} 0 \% \mathrm{~B} 2 \% \mathrm{D} 0 \% \mathrm{~B} 8 \% \mathrm{D} 0 \% \mathrm{~B} 7$

Гарбовский, Н. (2008). Dictionnaire russe-français des termes militaires. Moscou

Гаршин, И. (2012). Этимологический словарь Семёнова. Site internet: http://www.slovorod.ru/etym-semenov/sem-l.htm

Кузнецов, С (2014). Большой толковый словарь русского языка. Consulté le mai 30, 2020, site internet: http:/gramota.ru/slovari/info/bts/

Фасмер, М. (2019). Этимологический словарь русского языка (M. Р. Фасмер). Consulté le 9 juin, 2021, site internet: http://rus-yaz.niv.ru/doc/ etymological-dictionary/fc/slovar-203-4.htm\#zag-6891

Шанский, Н. (2019). Этимологические онлайн-словари русского языка. Site internet: https://lexicography.online/etymology/shansky/

Шапошников, А. (2010). Этимологический словарь Шапошникова. Consulté le mai 30, 2020, site internet: http://www.slovorod.ru/etym-shaposhnikov/ shap-b.htm 


\title{
TEACHING OF THE FRENCH LANGUAGE BY HOMONYMIE IN THE CASE OF FRENCH-RUSSIAN-LITHUANIAN MILITARY TERMS
}

\author{
Dr. Miroslav Stasilo
}

Vilnius University

\begin{abstract}
Summary
This article analyzes the teaching and learning of French as a foreign language in the Lithuanian Military Academy by focusing on the similarity and dissimilarity of professional military terminology in French, Russian and Lithuanian. Based on the corpus drawn from different specialized dictionaries, the article presents the variety of uses of French homonyms whose use in Lithuanian and Russian is differentiated and associated by focusing on the etymology and semantic values that they acquire in modern French-Lithuanian-Russian military discourse. The results of this research showed that the use of military homonyms is more common in Lithuanian and Russian than in French and Lithuanian. This study also presented a great complexity of disambiguation in current military discourse and its importance in the teaching of French at the academy.
\end{abstract}

Keywords: lexical analysis, learning, disambiguation, military discourse, military terms. 


\section{AUTORIAUS LYDRAŠTIS}

Autoriaus vardas, pavardė: Miroslav Stasilo

Mokslo laipsnis ir vardas: filologijos mokslų daktaras

Darbo vieta ir pareigos: Vilniaus universiteto Filologijos fakulteto Taikomosios kalbotyros institutas

Autoriaus mokslinių interesų sritys: užsienio kalbos, sociolingvistika, politikos mokslai

Telefonas ir el. pašto adresas: +37061144811 ;

francaisprancuzas@yahoo.fr

\section{AUTHOR'S COVER LETTER}

Author's name and surname: Miroslav Stasilo

Academic degree and name: Doctor of Linguistics

Workplace and position: Faculty of Philology, Applied Linguistics Institute, Vilnius University

Author's research interests: foreign language, sociolinguistics, political science

Telephone and e-mail address: +37061144 811;

francaisprancuzas@yahoo.fr 